\title{
Zur Rolle des Todes in der Heroisierung antiker Dichter
}

Stefan Tilg

\section{Heroisierung durch Dichter und von Dichtern}

Dichter sind die archetypischen Heldenmacher. Ohne Homer wäre Achill nicht der Achill, den wir kennen; ohne Vergil wäre Aeneas nicht unser Aeneas. Seit Homer schon wird die mediale Heroisierungskraft der Dichtung auch von den Dichtern selbst reflektiert. Als Hektor im 6. Buch der Ilias Helena und Paris in ihrem Haus besucht, ${ }^{1}$ entschuldigt sich Helena für alles Leid, das sie verursacht hat und rechtfertigt es mit dem Gedanken, dass die epischen Figuren so „den späteren Menschen zum Gesang werden". ${ }^{2}$ Hier spiegelt sich offenbar Homers eigene Funktion als epischer Sänger, genauso wie die Sänger Phemios und Demodokos, die in Buch 1 und 8 der Odyssee von den Taten und vom Ruhm der trojanischen Helden singen, oft als Spiegelbild Homers verstanden werden. Man könnte diesen Beispielen unzählige weitere aus der Weltliteratur hinzufügen. Das ist nicht das Ziel dieses Beitrags. Ich beschäftige mich hier nicht mit der Heroisierung durch Dichter, sondern mit der weniger offensichtlichen Heroisierung von Dichtern. Solche Heroisierungen können entweder von anderen oder als Selbstheroisierungen von den Dichtern vorgenommen werden. Vielleicht bauen diese Heroisierungsvorgänge auch auf der eingangs erwähnten archetypischen Funktion der Dichter als Heldenmacher auf: Weil die Dichter Glanz verleihen, kann dieser Glanz auf sie zurückfallen; weil sie Heroisches produzieren, haben sie selbst daran teil.

Heroisierungen von Dichtern sind an sich nichts Neues und Überraschendes. Im SFB 948 „Helden - Heroisierungen - Heroismen“ wurde die Rolle von Dichtern als Subjekt und Objekt von Heroisierungen bereits von Ann-Christin Bolay in ihrem Buch über Dichter und Helden im George-Kreis beschrieben. ${ }^{3}$ Der Standardfall, die Heroisierung anderer durch einen Dichter liegt etwa vor, wenn der Dichter und Literaturwissenschaftler Friedrich Gundolf (1880-1931) in seiner CaesarBiographie Caesar heroisiert. Die Heroisierung eines Dichters durch andere haben wir vor uns, wenn derselbe Friedrich Gundolf Goethe zum Helden stilisiert. Schließlich war ein Hang zur Selbstheroisierung den meisten Dichter-Mitgliedern des George-Kreises dadurch eigen, dass sie etwas Heldenhaftes in ihrer Fähigkeit sahen, Helden in ihrer epochalen Größe als Helden zu begreifen. Sie stilisierten

1 Hom. Il. 6, 318-368. Die Abkürzungen antiker Autoren und ihrer Werke folgen den Vorgaben in Hubert Cancik u. a. (Hg.): Der Neue Pauly, Bd. 1, Stuttgart 1996, S. XXXIX-XLVII.

2 Hom. Il. 6, 368.

3 Ann-Christin Bolay: Dichter und Helden. Heroisierungsstrategien in der Biographik des George-Kreises, Würzburg 2017. 
sich in diesem Sinn zu ,Haltungs-Helden`. All diese Heroisierungsprozesse funktionieren grundsätzlich auch ohne Heldentod und Tod des Helden. In diesem Beitrag möchte ich die Heroisierung von Dichtern dagegen dezidiert unter dem Aspekt des Todes der Dichter untersuchen. Als spezifisch antiker (eigentlich griechisch-antiker) Zugang bietet sich dafür der Heroenkult von Dichtern an, der an den Tod der Dichter gebunden ist und der mit einem Totenkult eng verwandt ist. Literarisch und in individuellen Praktiken wirkt diese Form der Dichterheroisierung in Rom, letztlich aber sogar bis heute nach. Ich vertrete die These, dass ohne die antiken Grundlagen und die schon damals vollzogene Entwicklung vom eigentlichen (religiösen) Dichterkult zum uneigentlichen (literarischen, metaphorischen), Dichterkult ${ }^{6}$ die Rolle des Todes in der Heroisierung von Dichtern bis zum heutigen Tag nicht recht verständlich wäre. Das den Tod voraussetzende Nacbleben von Dichtern aller Zeiten ist ja bis heute ein Nachweis ihrer Größe, ihrer Bedeutung und ihres Heldenstatus.

Wohlgemerkt: Hier handelt es sich eher um eine Variante des ,Todes des Helden' als um einen mit Opfer und Tat verbundenen ,Heldentod', in dem sich Heldentum symbolhaft verdichtet. Die in Viten und anderen Quellen berichteten Todesarten diverser antiker Dichter eignen sich selten für ein aktives Heldentum, wie wir es für einen ,Heldentod' nach heutigen Maßstäben gerne hätten. ${ }^{4}$ Von Homer wurde etwa berichtet, dass ein Orakel ihm den Tod prophezeite, wenn er einmal nicht länger mehr als andere Sterbliche erkennen könne. Genau das passiert dann, als ihm spielende Jungen ein Rätsel geben, das er nicht lösen kann. Er erinnert sich an das Orakel und hat gerade noch Zeit, sein eigenes Grabepigramm

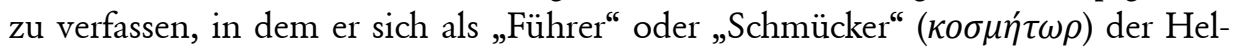
den sowie als göttliche Figur stilisiert (was schon einen frühen Beleg für die - hier zugeschriebene - literarische Selbstheroisierung eines Dichters darstellt). ${ }^{5}$ Dann rutscht er auf dem schlammigen Weg aus, fällt unglücklich hin und stirbt. Das ist für unsere Begriffe sicher kein ,Heldentod'. Gleichzeitig kann die Episode von Homers Ende aber auch stellvertretend für eine Reihe ähnlicher Erzählungen über antike Dichter gelten, in denen der Dichtertod etwas Numinoses, Gottgewolltes hat. Dieses Numinose ist wiederum etwas, was die postume Verehrung beflügelt. Obwohl kein ,Heldentod', ist ein solcher, Tod des Helden' nicht zufällig und bedeutungslos, sondern macht die spezifische Verehrung des Helden erst möglich.

4 Mary R. Lefkowitz: The Lives of the Greek Poets, Baltimore ${ }^{2} 2012$.

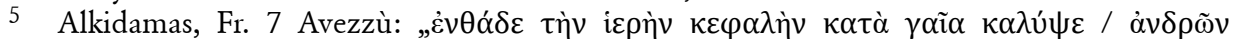

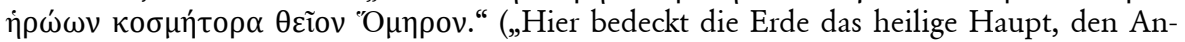
führer/Ausschmücker der Heroen, den göttlichen Homer.") Soweit nicht anders angegeben, stammen die deutschen Übersetzungen vom Verfasser. 


\section{Heroisierung von Dichtern im Heroenkult}

Ein berühmtes, L'Apothéose d'Homère (Homère déifié) betiteltes Gemälde des französischen Malers Jean-Auguste-Dominique Ingres (1780-1867) stellt ganz offensichtlich eine Heroisierung Homers dar (Abb. 1). Homer thront im Zentrum des Bildes und wird von einer geflügelten allegorischen Figur (Victoria oder das Universum) gekrönt. Als Archeget der Künste und des Wissens wird er von antiken und modernen Dichtern (z. B. Pindar und Shakespeare), Malern (z. B. Phidias und Michelangelo), Musikern (z. B. Orpheus und Mozart), Rednern und Historikern (z. B. Demosthenes und Herodot), Philosophen und Gelehrten (z. B. Platon und Aristarch) sowie Staatsmännern (z. B. Peisistratos und Alexander dem Großen) umringt. Ingres bezeichnete diese „Heldenverehrer" explizit als „Homeriden“, was wörtlich eine Abstammung von Homer impliziert und womit hier Homer als geistiger ,Vater ${ }^{6}$ seiner ,Kinder ${ }^{6}$ in der Nachwelt erscheint. Auf dem Sockel, auf dem Homer sitzt und der von Allegorien der Ilias und der Odyssee flankiert ist, liest man

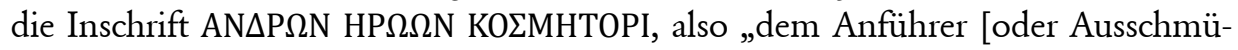
cker] der Heroen “. ${ }^{6}$ Im Hintergrund sieht man einen Homer geweihten Tempel.

Man könnte das alles als Ingres' persönliche Spielerei mit Motiven und Begrifflichkeiten des Heroischen interpretieren, wenn nicht so viel antike Realität dahinter zu finden wäre. An erster Stelle muss hier ein antikes Relief aus dem 2. Jahrhundert v. Chr. genannt werden, das die mehr oder weniger direkte Vorlage für Ingres darstellte und das vom niederländischen Altertumswissenschaftler Gisbert Cuper (1644-1716) Apotheose des Homer genannt wurde (Abb. 2). ${ }^{7}$ Der Künstler, ein gewisser Archelaos von Priene, nennt sich in der Signatur des Reliefs selbst, ist aber sonst nicht bekannt. Es handelt sich bei der Darstellung auf dem Relief nach gängiger Interpretation um die Weihegabe eines anonymen Dichters (ganz rechts mit dem Dreifuß, einem typischen Weihegeschenk, dargestellt), der sie aus Dankbarkeit über den Sieg in einem musischen Wettkampf in Auftrag gab und einem Heiligtum stiftete. Die Szenerie stellt einen Musenberg dar. Ganz oben sind Zeus und Mnemosyne als die Eltern der neun Musen zu sehen. Die Musen selbst folgen dann auf den beiden darunterliegenden Ebenen zusammen mit ihrem Kithara spielenden Anführer Apollon. Auf der untersten Ebene thront schließlich Homer, von Allegorien der Welt (Oikumene) und der Zeit (Chronos) bekränzt und von Personifikationen der Ilias und der Odyssee flankiert. Rechts von ihm huldigen ihm mit einem Stieropfer Personifikationen des Mythos (Mythos), der Geschichte (Historia), der Dichtung (Poiesis), der Tragödie (Tragodia) und der Komödie

6 Die Wendung ist Homers vorgeblichem Grabepigramm entnommen, siehe oben Anm. 5.

7 Gisbert Cuper: Apotheosis vel consecratio Homeri, Amsterdam 1683; siehe jetzt auch Sitta von Reden: Neue Helden in der hellenistischen Polis. Der Dichterheros und sein Bild im Archelaosrelief von Priene, in: Achim Aurnhammer / Ulrich Bröckling (Hg.): Vom Weihegefäß zur Drohne. Kulturen des Heroischen und ihre Objekte (Helden - Heroisierungen Heroismen 4), Würzburg 2016, S. 41-58. Das Relief befindet sich heute in London, British Museum, Inv. 2191. 


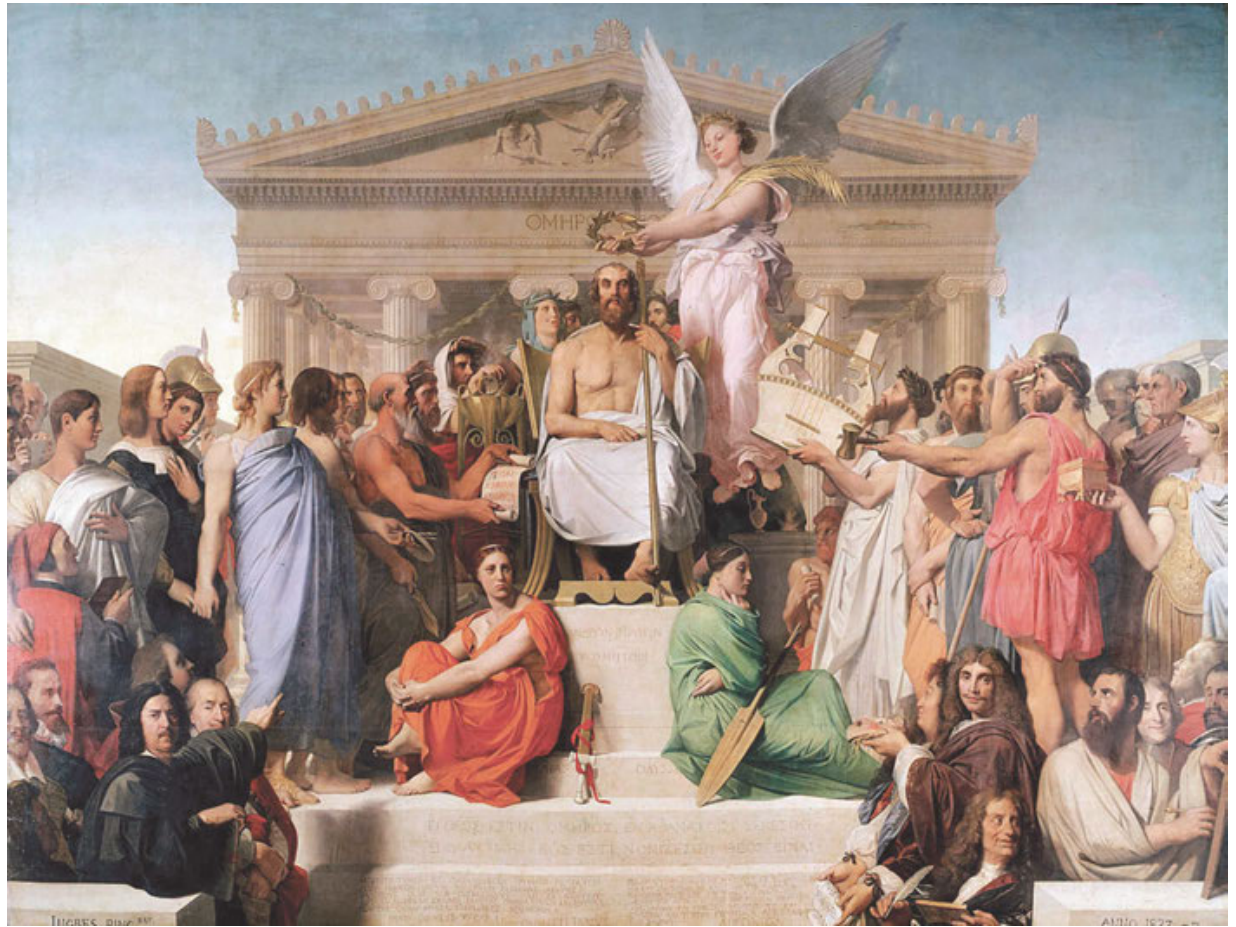

Abb. 1: Jean Auguste Dominique Ingres, L'Apothéose d'Homère (Homère déifié), Öl auf Leinwand, 1827, Paris, Musée du Louvre, Inv. 5417.

(Komodia), der Natur (Physis), der Tugend (Arete), des Gedächtnisses (Mneme), des Vertrauens (Pistis) und der Weisheit (Sophia). Obwohl die Bildkomposition bei Ingres eine andere ist, sind die Parallelen bezüglich Homer - Thron, Bekränzung, flankierende Allegorien von Ilias und Odyssee, Huldigung und Verehrung als Quell der Künste und des Wissens - unübersehbar. Das Stieropfer führt uns im Fall des antiken Reliefs noch deutlicher in die Welt des realen griechischen Kults, in dem neben anderen Heroen auch Dichter wie Homer verehrt wurden. Gisbert Cuper, der das Relief erstmals untersuchte, nahm es deshalb zum Anlass, auch die zu seiner Zeit verfügbaren Zeugnisse für die kultische Heroisierung von Dichtern in der griechischen Antike zu sammeln. In unserer Zeit wurde Cupers Arbeit vor allem von Diskin Clay fortgeführt, der ausgehend von Neufunden zum Kult des Dichters Archilochos das allgemeine Thema der griechischen Dichterkulte wieder aufrollte. ${ }^{8}$

Gehen wir aber zuerst noch einen Schritt zurück. Worum geht es beim griechischen Heroenkult und was ist ein „Heros` überhaupt?? Das Wort „Heros' konnte

8 Diskin Clay: Archilochos Heros. The Cult of Poets in the Greek Polis, Washington, DC 2004.

9 Eine gute Übersicht über Formen und historische Entwicklungen gibt Christopher P. Jones: New Heroes in Antiquity, Cambridge, MA 2011; siehe auch Ralf von den Hoff u. a.: Das 


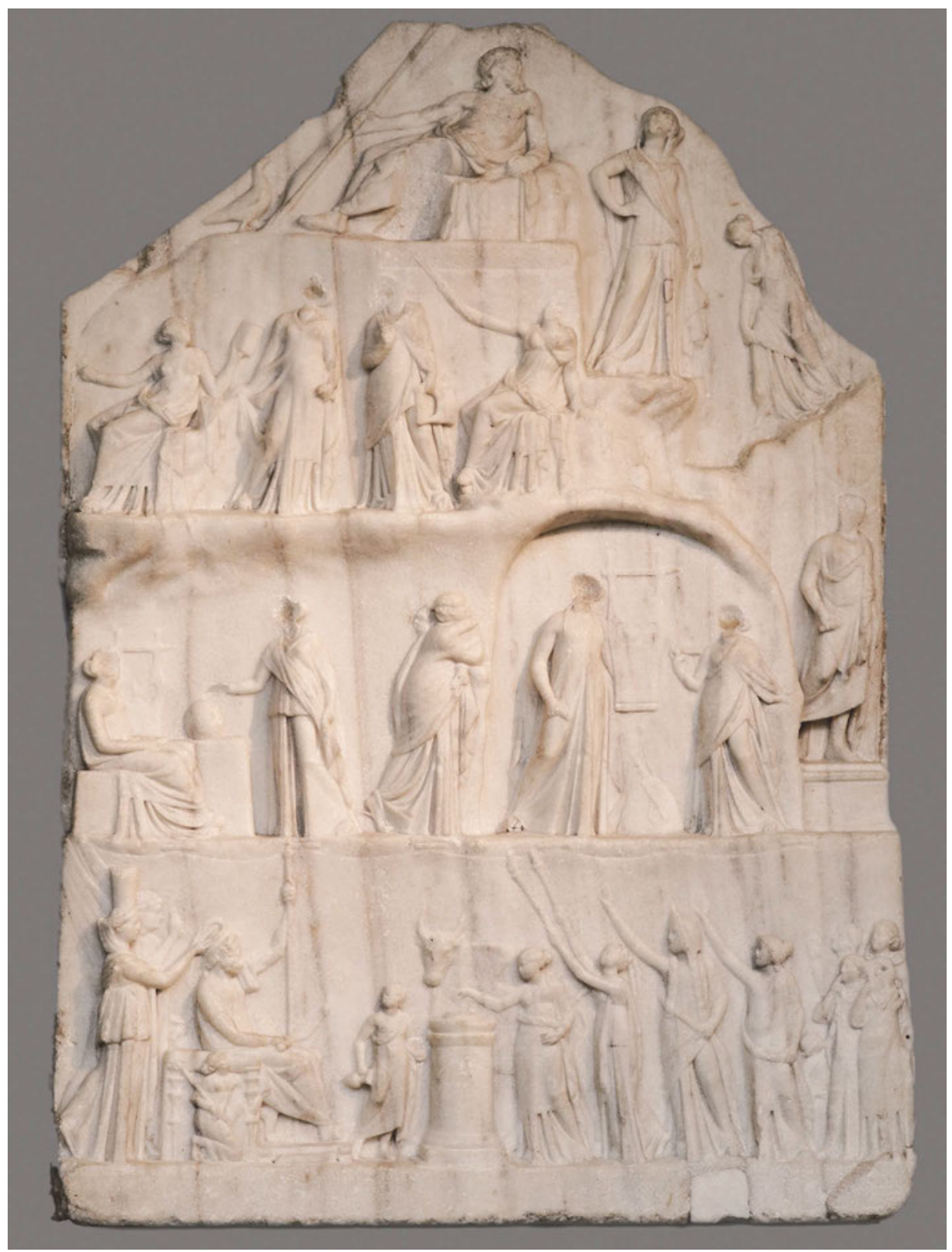

Abb. 2: Archelaos von Priene, Apotheose des Homer, Marmor, 3. Jh. v. Chr., London, British Museum, Inv. 1819,0812.1.

Heroische in der neueren kulturhistorischen Forschung. Ein kritischer Bericht, in: H-SozKult, Kapitel 4.1. Antike, 28. Juli 2015, www.hsozkult.de/literaturereview/id/forschungs berichte-2216, 14. November 2018. 
in der griechischen Antike sowohl literarisch als auch kultisch verstanden werden. Literarisch bezeichnete es die Figuren der homerischen (und später anderer) Epen, kultisch meinte es verstorbene Personen, denen besondere Verehrung zuteilwurde und die man sich zwischen Menschen und Göttern vorstellte. Kultische Heroen waren materiell und lokal an Gräber, besondere Orte, Heiligtümer u. Ä. gebunden. Man verehrte sie, um sich ihres Beistands zu versichern, um ihren Zorn abzuwenden, aber auch eines gewissen symbolischen Kapitals wegen, über das eine Gemeinschaft verfügte, wenn sie das Heiligtum dieses oder jenes bekannten Heros besaß. Grundzüge dieser Verehrungsmuster sind auch heute noch bekannt, am besten wohl aus dem christlich-katholischen Heiligenkult, der manchmal im Sinn einer Kultkontinuität als Fortsetzung des griechischen Heroenkults gedeutet wird. Kultisch heroisiert werden konnten ,große ${ }^{\mathbf{c}}$, in ganz Griechenland bekannte literarisch-epische Helden wie z. B. Achilles, dessen vermeintliches Grab in der Troas sich zu einer Pilgerstätte entwickelte, dem aber auch an anderen Orten Kulte gewidmet waren - spätestens nach Homer vermischten sich literarische und kultische Heroen; manche Forscher wollen schon bei Homer Reflexe eines historischen Heroenkults erkennen. ${ }^{10}$ Kultisch verehrt werden konnten auch ,kleine', lokale Helden, die allerdings ebenso unhistorisch wie die großen panhellenischen waren. Ein Beispiel dafür wäre etwa ein gewisser Heros Androkrates, den Herodot und Thukydides im Kontext ihrer Berichte über die böotische Stadt Plataiai als mythischen Gründervater der Stadt erwähnen und dessen Heiligtum (Heroon) sie nennen. ${ }^{11}$ Schließlich konnten aber auch historische Personen kultisch verehrt werden. Diese Personen waren entweder in einer Familie besonders bedeutsam (z. B. als Stammväter; hier gibt es eine Schnittstelle zum ,gewöhnlichen' Grab- und Totenkult) oder sie hatten wegen ihrer besonderen körperlichen oder geistigen Leistungen ein öffentliches Interesse. So konnten z. B. Athleten, Philosophen und - Dichter heroisiert werden.

Dichterkulte waren also Teil des allgemeinen griechischen Heroenkults. Laut Clay war der vielseitige, späteren Generationen aber vor allem als der Erfinder aggressiver Spottdichtung bekannte Archilochos von Paros (7. Jahrhundert v. Chr.) der älteste Dichter, dem eine solche Verehrung zuteilwurde. Vielleicht spielte es bei dieser ersten Heroisierung eines Dichters eine Rolle, dass Archilochos in Paros und der parischen Kolonie Thasos nicht nur als Dichter, sondern auch als Politiker und Soldat aktiv und mit den Polisgeschäften eng verbunden war. Vielleicht wurde in seinem Fall also praktisch-tathaftes Heldentum, als das ursprüngliche, auf dichterisches Heldentum übertragen. Später war diese Engführung von Tatheld und Dichterheld nicht mehr nötig. Auch lief Homer Archilochos bald

10 Prominent vertritt letztere Position z. B. Gregory Nagy in seinen zahlreichen Werken zu Homer und homerischem Heldentum. Eine Art Kondensat dieses Oeuvres ist sein Kapitel The Epic Hero, in: John M. Foley (Hg.): A Companion to Ancient Epic, Malden, MA 2005, S. 71-89.

11 Hdt. 9, 25, 3; Thuk. 3, 24, 1. 
den Rang als der meistheroisierte antike Dichter ab. Weitere Dichter wie Pindar und Aischylos kamen bald hinzu, mit Sappho und Korinna auch zwei Dichterinnen (Sappho ist übrigens die einzige weibliche Homer-Verehrerin auf Ingres' Gemälde). Clay identifiziert insgesamt 36 Orte der griechischen antiken Welt von der Magna Graecia über Athen bis zur Schwarzmeerküste -, an denen Dichter kultisch verehrt wurden. ${ }^{12}$

Wie manifestierten sich solche Kulte? Zum Beispiel durch Heiligtümer, Opfer und Inschriften, wie es für Archilochos in der sog. Mnesiepes-Inschrift aus dem 3. Jahrhundert v. Chr. belegt ist. Diese erst 1949 auf Paros entdeckte und in den 1950er Jahren publizierte Inschrift ist eine der Hauptquellen für Archilochos' Kult auf seiner Heimatinsel. Verfasst und geweiht wurde sie von einem gewissen Mnesiepes, der in der Inschrift von Orakeln des delphischen Apollon an ihn berichtet. Apollon hätte Mnesiepes demgemäß aufgetragen, einen Archilochos-Kult in einem „Archilocheion“ genannten heiligen Bezirk einzurichten. Mnesiepes schreibt dazu in der 1. Person Plural:

Da nun Apollon diese Dinge geoffenbart hat, nennen wir den Ort „Archilocheion“ und wir errichten Altäre und wir opfern sowohl den Göttern als auch Archilochos und wir ehren ihn gemäß der Antwort des Gottes. Was aber den Inhalt der Inschrift angeht, die wir planten, so wurde er uns von den Alten überliefert [...]. ${ }^{13}$

Als Inhalt der Weiheinschrift wird dann eine Legende erzählt, wonach dem Knaben Archilochos, als er eine Kuh zum Markt brachte, die Musen erschienen seien und ihn zum Dichter beriefen. Diese Legende finden wir bildlich dargestellt auf einer Büchse (Pyxis) aus Eretria auf Euböa (ca. 460/450 v. Chr.). ${ }^{14}$ Auch bildliche Darstellungen wie diese oder wie das Relief des Archelaos von Priene konnten Manifestationen eines Dichterkults sein. Eine andere Form von Dichterkult, in dem die Performanz des jeweiligen Werks des Dichters eine Rolle spielt, ist uns z. B. in der Aischylos-Vita bezeugt:

Als er [Aischylos] starb, ehrten ihn die Einwohner von Gela mit einem prachtvollen Begräbnis auf ihrem Friedhof. Auf sein Grabmonument schrieben sie: „Dieses Denkmal bedeckt Aischylos [...]“. Professionelle tragische Schauspieler besuchten sein Grabmonument und opferten ihm dort und führten seine Tragödien auf. ${ }^{15}$

12 Clay: Archilochos Heros (Anm. 8), S. 5 mit Karte.

13 Hier wiedergegeben nach der Edition bei Clay: Archilochos Heros (Anm. 8), S. 104-110:

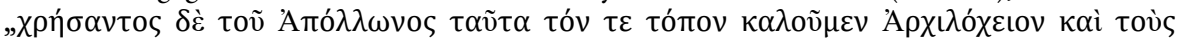

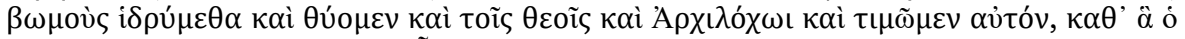

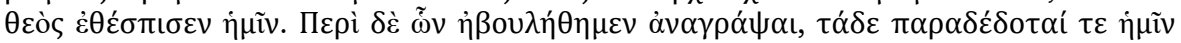

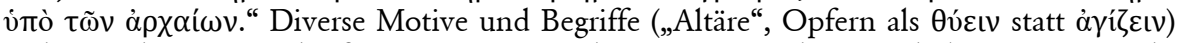
widersprechen einer scharfen Trennung zwischen Götter- und Heroenkult, wie sie manchmal behauptet wird. Clay stellt eine solche Verwischung der Sphären des Göttlichen und des Heroischen öfter fest.

14 Attisch-weißgrundige Pyxis, Boston, Museum of Fine Arts, Inv. 98.887: Beazley Archive Data Base Vase No. 209554: http://www.beazley.ox.ac.uk, 14. November 2018.

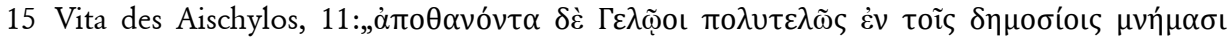

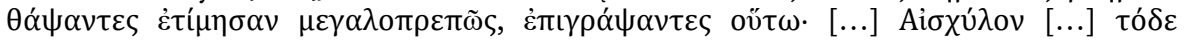


Passend für einen Tragödiendichter wurde Aischylos demnach an seinem Grab mit der Aufführung seiner Stücke geehrt. Schließlich manifestierten sich Dichterkulte auch in Tempeln, Statuen und Münzprägungen. Alle diese Elemente werden in einem kurzen Bericht des Geographen Strabon angesprochen, der in augusteischer Zeit lebte und in seinen Geographika ${ }^{16}$ die kleinasiatische Stadt Smyrna beschreibt. Smyrna galt (neben anderen Städten) als Geburtsort Homers und pflegte deshalb einen Homer-Kult:

[In Smyrna] gibt es sowohl eine Bibliothek als auch ein Homer-Heiligtum (Homereion), eine rechteckige Stoa mit einem Tempel Homers und einer Statue [...] sogar eine Bronzemünze heißt bei ihnen „Homereion“. ${ }^{17}$

Ingres' Homer-Tempel im Hintergrund seiner Apothéose d'Homère ist also keine bloße Fantasie. Es gab einen solchen Tempel zumindest in Smyrna. Darüber hinaus sind Münzen, die ein Abbild Homers zeigen, aus Smyrna und anderen Städten archäologisch bezeugt. ${ }^{18}$

\section{Vom Kult über die Literatur zur Selbstheroisierung}

Alle bisherigen Beispiele von Dichterkulten fallen in die Kategorie der Heroisierung von Dichtern durch andere. Selbstheroisierungen waren nicht darunter. Das ist kein Zufall, denn die reale Kultpraxis schließt eine Heroisierung zu Lebzeiten und durch sich selbst eigentlich aus. Einen Ansatz für Letzteres kann man aber vielleicht - wenn Clays Interpretation zutrifft - für den Fall des Antigonos von Knidos im 3. Jahrhundert v. Chr. ausmachen. Antigonos war ein Dichter, der uns nur durch ein ihm gewidmetes Heroon in der Nekropole der kleinasiatischen Stadt Knidos bekannt ist. Beim Betreten des Heroons lesen die Besucher in einer Inschrift u. a. folgende vom Götterboten Hermes (der sich am Ende selbst nennt) gesprochene Worte:

Noch ein Stückchen Weg ist übrig. Doch du wirst zur Höhe gelangen, wenn du dem kurzen Weg zu meiner Linken folgst, Fremder, und wenn du mich gegrüßt hast, trittst du ein. Du wirst den heiligen Bezirk des wohlwollenden Heroen Antigonos betreten. Wenn dir die Musen eine Gabe gegeben haben, dann opfere den Göttern dieses Orts etwas von deiner Kunstfertigkeit. Für Sänger ist eine Bühne und ein Schrein errichtet unter dem Berg $[\ldots] .{ }^{19}$

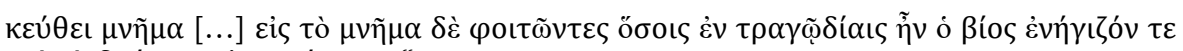

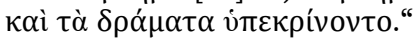

16 Strab. 14, 1, 37.

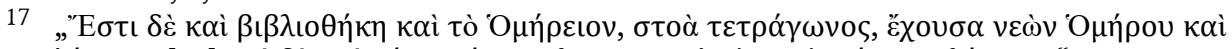

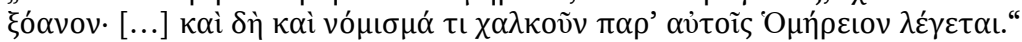

18 Abbildungen z. B. bei Paul Zanker: Die Maske des Sokrates. Das Bild des Intellektuellen in der antiken Kunst, München 1995, S. 159-160, Abb. 87-88.

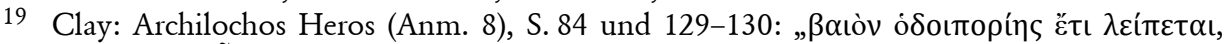

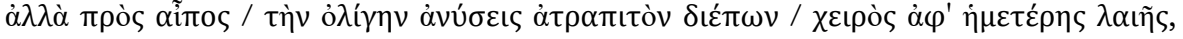


Wir finden hier typische Elemente eines Dichterkults. Antigonos wird als Heros angesprochen. Er hat einen heiligen Bezirk. Das im Heiligtum zu bringende Opfer scheint hier schon metaphorisch gedacht. Es handelt sich um musische Darbietungen, die an sich selbst allerdings wieder typisch für Dichterkulte sind - siehe das Beispiel von Aischylos oben. Besonders an Antigonos' Heroon ist aber, dass sich der Dichter hier vielleicht selbst ein Denkmal gesetzt hat - jedenfalls schließt Clay aus dem Fehlen von Hinweisen auf einen Stifter und aus der sonstigen Obskurität von Antigonos, dass die Inschrift von ihm selbst stammte und er also schon zu Lebzeiten für seine Heroisierung nach dem Tod vorgesorgt habe.

Im Folgenden möchte ich dieser Figur der Selbstheroisierung durch die imaginäre Antizipation des eigenen Todes weiter nachgehen. In der realen Kultpraxis haftet ihr etwas Blasphemisches an und sie ist dementsprechend selten zu finden. Sie tritt dort prominenter literarisch hervor, wo die kultische Praxis zurücktritt und ,nur' noch als Bildspender dient. In der klassisch-antiken Welt war das bei den Römern der Fall, die keinen Heroenkult wie die Griechen kannten. Die Römer hatten gar keinen eigenen Begriff für ,Heros` - wenn sie einen Fachterminus brauchten, benutzten sie den griechischen. Natürlich wurden auch in der römischen Welt Dichter verehrt, aber diese Verehrung war individueller und literarischer als in den griechischen Dichterkulten. Das bekannteste Beispiel ist die quasikultische Verehrung, die Vergil von seinen epischen Nachfolgern Silius Italicus und Statius gegen Ende des 1. Jahrhunderts n. Chr. erfuhr. Plinius der Jüngere berichtet in seinem Nekrolog auf Silius Italicus über dessen Villen und kommt davon ausgehend auf Vergil zu sprechen:

Überall gab es viele Bücher, viele Statuen, viele Bilder, die er [Silius Italicus] nicht nur besaß, sondern geradezu verehrte, vor allen andern das Vergils, dessen Geburtstag er feierlicher beging als seinen eigenen, meist in Neapel, wo er dessen Grabmal (monimentum) wie ein Heiligtum (ut templum) zu besuchen pflegte. ${ }^{20}$

Einen ähnlichen ,Grabkult ${ }^{\natural}$ pflegte Statius nach seiner eigenen Aussage in der Gedichtsammlung Silven: „Lässig zupfe ich mit dem Daumen die zarten Saiten, sitze am Rand der Gruft (templum) des Maro und fasse Mut und besinge das Grab des großen Meisters. “21

Das Vokabular („verehren“, „Grabmal“ als „Tempel“) lässt es plausibel erscheinen, dass solche individuellen und literarisch ausgestalteten Verehrungspraktiken von griechischer Kultpraxis angeregt wurden - kulturellen Austausch zwischen Rom und Griechenland gab es ja genug. Wenn die Kultpraxis aber nicht mehr

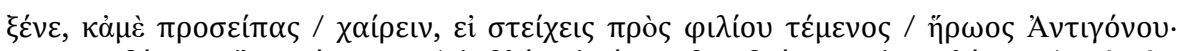

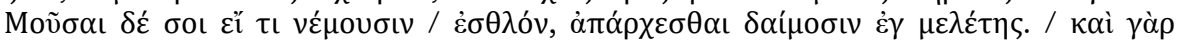

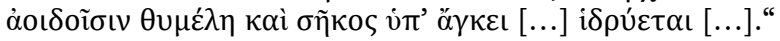

Plin. epist. 3, 7, 8: „Multum ubique librorum, multum statuarum, multum imaginum, quas non habebat modo, verum etiam venerabatur, Vergili ante omnes, cuius natalem religiosius quam suum celebrabat, Neapoli maxime, ubi monimentum eius adire ut templum solebat."

21 Stat. silv. 4, 4, 53-55: „Tenues ignavo pollice chordas / pulso Maroneique sedens in margine templi / sumo animum et magni tumulis adcanto magistri.“ 
wörtlich verstanden wird, kann sie - literarisch und individuell - auch für ,Selbstheroisierungen' Pate stehen. Einen solchen Fall möchte ich in Horaz' berühmter Schlussode seiner ursprünglich drei Bücher umfassenden Odensammlung entdecken. Hier blickt der Dichter auf seine literarische Leistung zurück und setzt sich selbst ein Denkmal dafür:
Ich habe ein Monument/Grabmal (monumentum) errichtet, das Erz überdauert, das den majestätischen Bau der Pyramiden überragt, das nicht der nagende Regen noch der Nordwind zügellos zu zerstören vermag, nicht die unzählbare Folge der Jahre und die Flucht der Zeit. Nicht gänzlich werde ich sterben - ein großer Teil von mir wird der To- desgöttin entgehen. Unaufhörlich und jugendfrisch im Ruhm werde ich in der Nachwelt wachsen, solange der Priester mit der schweigenden Jungfrau auf das Kapitol steigen wird. Nennen wird man mich, wo der Aufidus heftig tost und wo Daunus, an Wasser arm, über ländliche Völker geherrscht hat. ${ }^{22}$ Aus niederem Stand mächtig geworden, ha- be ich als erster den äolischen Sang in italische Weisen überführt. Greife den stolzen Preis, der dem Verdienst gebührt, und bekränze mir gnädig, Melpomene, mit delphi- schem Lorbeer das Haar. ${ }^{23}$

Horaz' Spiel mit Grabmetaphorik ist unverkennbar. Der Dichter hat sich nicht nur selbst ein Grab-/Denkmal errichtet, er will damit auch noch die als Grabmäler bekannten Pyramiden übertreffen. Der Tod, die Überwindung des Todes durch das dichterische Werk und der postume Ruhm sind das zentrale Thema der Ode. Am Ende steht, wie in Ingres' und Archelaos' Apotheosen des Homer, eine triumphale Bekränzung durch ein höheres Wesen (hier die Muse Melpomene im Verein mit dem delphischen Apollon). Der Lorbeer, mit dem sich Horaz bekränzen lässt, war ursprünglich das Attribut der Sieger in den delphischen Sportwettkämpfen (Pythien), in Rom aber auch jenes der erfolgreichen Feldherrn, wenn sie im Triumph nach Rom einzogen. Michael Putnam hat in seinem Aufsatz The Lyricist as Hero den triumphalen Gestus von Horaz als Sieger hervorgehoben und einige weitere ,heroische' Facetten dieser Ode benannt, in diesem Kontext aber nicht auf die Bildlichkeit von Grab und Tod hingewiesen. ${ }^{24}$ Diese Bildlichkeit ist meines Erachtens aber untrennbar mit der besonderen Art von Heroisierung verbunden, die Horaz vorschwebt und die er vielleicht in direkter Anlehnung an den griechischen Heroenkult konzipiert hat. Hier wie dort ist der

22 Horaz spielt hier auf seine süditalische Heimat an. Der Aufidus, heute Ofanto, ist der Hauptfluss Apuliens. Daunus ist ein legendärer apulischer König. Er ist „an Wasser arm“ und herrscht „,̈̈ber ländliche Völker“, weil Apulien damals für häufige Dürren und für seine bäuerliche Bevölkerung bekannt war.

23 Hor. carm. 3, 30: „Exegi monumentum aere perennius / regalique situ pyramidum altius, / quod non imber edax, non aquilo impotens / possit diruere aut innumerabilis / annorum series et fuga temporum. / non omnis moriar multaque pars mei / vitabit Libitinam: usque ego postera / crescam laude recens, dum Capitolium / scandet cum tacita virgine pontifex: / dicar, qua violens obstrepit Aufidus / et qua pauper aquae Daunus agrestium / regnavit populorum, ex humili potens / princeps Aeolium carmen ad Italos / deduxisse modos. sume superbiam / quaesitam meritis et mihi Delphica / lauro cinge volens, Melpomene, comam.“

24 Michael C. J. Putnam: Horace C. 3. 30. The Lyricist as Hero, in: Ramus 2, 1973, S. 1-19. 
Tod Bedingung für die Verehrung durch eine Gemeinschaft, hier wie dort knüpft sich die Verehrung an ein ,Grab'. Eine ganz ähnliche Selbstheroisierung nahm nach Horaz und in direktem Anschluss an ihn auch Ovid am Ende seiner Metamorphosen vor:

Nun habe ich ein Werk vollendet, das nicht Jupiters Zorn, nicht Feuer, nicht Eisen, nicht das nagende Alter wird vernichten können. Wann er will, mag jener Tag, der nur über meinen Leib Gewalt hat, meines Lebens ungewisse Frist beenden. Doch mit meinem besseren Teil werde ich fortdauern und mich hoch über die Sterne emporschwingen; mein Name wird unzerstörbar sein, und so weit sich die römische Macht über den unterworfenen Erdkreis erstreckt, werde ich vom Mund des Volkes gelesen werden und, sofern an den Vorahnungen der Dichter auch nur etwas Wahres ist, durch alle Jahrhunderte im Ruhm fortleben (vivam). ${ }^{25}$

Hier fehlt zwar das in Bezug auf den griechischen Heroenkult wichtige Element des Grabes; dafür haben wir aber wieder die Idee des Kontakts mit dem Überirdischen (dort die Muse und Apollon, hier die Sterne) und der bleibenden Erinnerung. Wie bei Horaz ist der Tod Bedingung der Verehrung. Erst im Tod beginnt das heroische ,Nachleben' des Dichters (passenderweise ist das letzte Wort vivam, „ich werde leben“). ${ }^{26}$ Charles Segal sieht das Heroische in diesen Schlussversen vor allem in der trotzigen Selbstbehauptung des Dichters Ovid angesichts seiner Verbannung durch Augustus, der wohl mit „Jupiters Zorn“ angesprochen ist. Das von Segal nicht beachtete Todesmotiv hat aber, wie ich zeigen wollte, auch seine eigene Heroik.

\section{Fazit und Ausblick}

Ich habe in diesem Beitrag mit dem Gedanken gespielt, dass reale griechische Dichterkulte eine Inspiration für literarische Heroisierungen und Selbstheroisierungen römischer Dichter waren. Die zentrale Gemeinsamkeit ist die heroisierende Kraft des Todes: Diese entrückt die verehrten Dichter in eine überirdische Sphäre, die ihnen eine numinose Qualität verleiht und so einen bleibenden Erinnerungskult möglich macht. Eine solche Inspiration scheint mir aufgrund der verwendeten Begriffe und Motive sowie des intensiven Kulturkontakts zwischen griechischer und römischer Welt plausibel. Schlagend beweisen lässt sie sich nicht. Man muss sich aber auch gar nicht auf eine Abhängigkeit römischer Dichter von griechischen Dichterkulten festlegen, um den typologischen Gemeinsamkeiten im Hinblick auf das Thema ,Heldentod' und ,Tod des Helden“ etwas ab-

25 Ov. met. 15, 871-879: „Iamque opus exegi, quod nec Iovis ira nec ignis / nec poterit ferrum nec edax abolere vetustas. / cum volet, illa dies, quae nil nisi corporis huius / ius habet, incerti spatium mihi finiat aevi; / parte tamen meliore mei super alta perennis / astra ferar, nomenque erit indelebile nostrum; / quaque patet domitis Romana potentia terris / ore legar populi, perque omnia saecula fama / (siquid habent veri vatum praesagia) vivam."

Charles Segal: Ovid. Metamorphoses, Hero, Poet, in: Helios 12.1, 1985, S. 49-63. 
zugewinnen. Die griechischen und römischen Beispiele können gemeinsam vor einer Unterschätzung des ,Todes des Helden' als rein zufälligem Element in Heroisierungsprozessen warnen. In allen Beispielen ist der Tod die conditio sine qua non der Heroisierung. ${ }^{27}$ Der Tod verleiht den Heroen eine besondere numinose Qualität und führt zu einem Kult, sei er nun mehr religiöser oder literarischer Natur (wobei die literarischen Beispiele in ihrer Begrifflichkeit und Bildlichkeit eine religiöse Komponente evozieren).

Martial (ca. 40-104 n. Chr.) schreibt in einem seiner Epigramme an einen gewissen Vacerra scherzhaft: „Nur die Alten bewunderst du, Vacerra, und lobst allein die toten Dichter. Verzeih', ich bitte dich, Vacerra: So viel ist's mir nicht wert zu sterben, nur um dir zu gefallen." ${ }^{28}$ Hinter aller Ironie ist hier auch ein Körnchen Wahrheit zu finden. Tote Dichter werden bis heute anders, intensiver, ,kultischer' als lebende verehrt. Viele von uns haben wohl schon ein Dichtergrab besucht. Jene, die es noch nicht getan haben oder weitere Besuche planen, können sich von Büchern wie Peter Andreas' Im letzten Garten. Besuch bei toten Dichtern führen lassen. ${ }^{29}$ Darin stellt der Autor von Petrarca $(\dagger 1374)$ bis zu Elias Canetti $(\dagger 1994)$ Fotografien von Dichtergräbern Textauszügen der jeweiligen Dichter gegenüber und ,heroisiert' sie damit in gewisser Weise über ihren Tod.

Streng genommen - so viel sollte der vorliegende Beitrag gezeigt haben - hätte Andreas aber mit der griechisch-römischen Antike anfangen müssen. Dichterkulte im eigentlichen, kultischen, und im uneigentlichen, literarisch-metaphorischen Sinn nahmen damals ihren Anfang und begründeten eine Tradition. Regelrechte Pilgerfahrten zum Grab Vergils in Neapel dienten sogar als unmittelbares Vorbild für ähnliche Praktiken in der Frühen Neuzeit, ${ }^{30}$ aus denen sich dann letztlich auch viel von unserer modernen Heroisierung toter Dichter erklärt. Diese Präsenz der Antike in späteren Heroisierungspraktiken spiegelt sich sinnfällig in einem der bekanntesten Gemälde von Vergils Grab. Es stammt vom englischen Maler Joseph Wright, der Neapel 1774 besuchte und 1779 Vergils dortiges Grab in stimmungsvollem Mondlicht darstellte (Abb. 3). Wright legte damit Zeugnis von einem ,Dichterkult' seiner Zeit ab, bezog aber auch die antike Verehrung Vergils mit ein, indem er Silius Italicus - wie von Plinius dem Jüngeren berichtet - am Grab Vergils studieren bzw. deklamieren lässt.

27 Das Problem der Verehrung toter Individuen als Helden überschneidet sich dabei mit dem des postumen Ruhms, vgl. dazu schon Julian Hirsch: Die Genesis des Ruhmes. Ein Beitrag zur Methodenlehre der Geschichte, Leipzig 1914, bes. S. 42-51 (im Inhaltsverzeichnis prägnant zusammengefasst mit dem Satz „Der Ruhm entsteht nicht nur nachdem, sondern: weil das Individuum tot ist").

Mart. 8, 69: „Miraris veteres, Vacerra, solos / nec laudas nisi mortuos poetas, / ignoscas petimus, Vacerra: tanti / non est, ut placeam tibi, perire."

29 Peter Andreas: Im letzten Garten. Besuch bei toten Dichtern. Mit einem Essay von Günter Kunert, Hildesheim 2005.

30 John B. Trapp: The Grave of Vergil, in: The Journal of the Warburg and Courtauld Institutes 47, 1984, S. 1-31. 


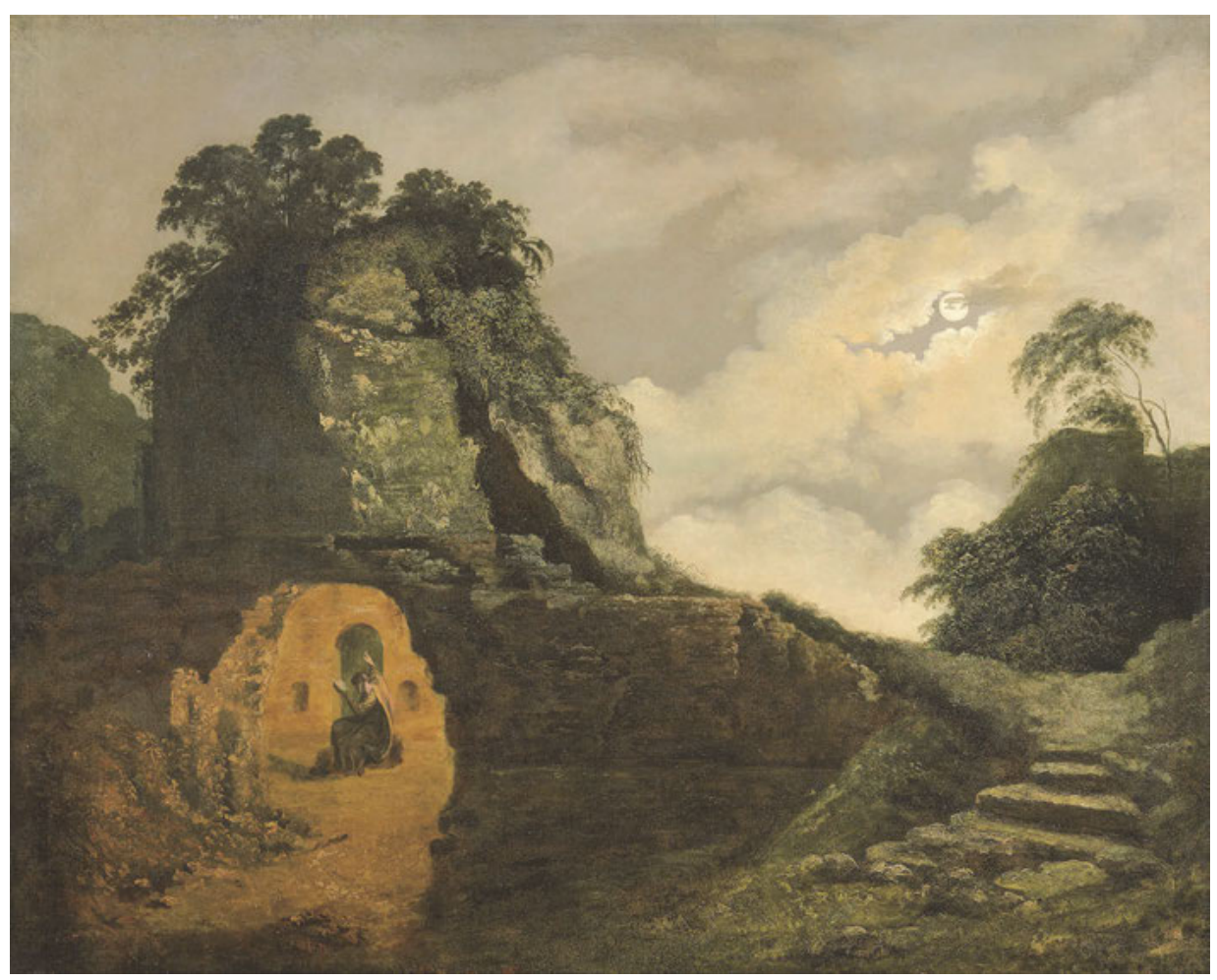

Abb. 3: Joseph Wright of Derby, Virgil's Tomb by Moonlight, with Silius Italicus, 1779, New Haven, Yale Center for British Art, Paul Mellon Collection, Inv. B1973.1.68.

\section{Abbildungsnachweise}

Abb. 1: wartburg.edu / Wikimedia Commons. Fotograf: Alonso de Mendoza, https://commons.wikimedia.org/wiki/File:Jean_Auguste_Dominique_In gres,_Apotheosis_of_Homer,_1827.jpg.

Abb. 2: Wikimedia Commons. Fotografin: Marie-Lan Nguyen, https://com mons.wikimedia.org/wiki/File:Apotheosis_Homer_BM_2191.jpg.

Abb. 3: Yale Center for British Art, http://collections.britishart.yale.edu/vu find/Record/1666634. 
